\section{Crowd control}

\section{T. Vicsek}

Department of Biological Physics, Eötvös University, Pázmány p. Stny 1A, H-1117 Budapest, Hungary

$T$ he application of ideas, methods and results of statistical $I$ physics to a wide range of phenomena occurring outside of the realm of the non-living world has gained a great momentum recently. Among many others, examples include the studies of various group activities of people from the physicist's viewpoint. Here, I shall give a partial account of some of the new investigations in this direction, involving the interpretation of such collective human activities as group motion and synchronisation.

On the small scale side of the size/complexity spectrum, in the world of atoms and molecules collective behaviour is also considered to be an important aspect of the observed . Furthermore, there are articles on collectively migrating bacteria, insects or birds and additional interesting results are published on phenomena in which groups of various organisms or non-living objects synchronise their signals or motion. This is the natural scientist's aspect of how many objects behave together. However, if you search for a collective behaviour related item with your web browser most of the texts popping up will be concerned with group activities of humans including riots, fashion or panics.

What is common in these seemingly diverse phenomena involving interpretations ranging from social psychology to statistical physics? The answer is that they happen in systems consisting of many similar units interacting in a relatively well defined manner. These interactions can be simple (attraction/repulsion) or more complex (combinations of simple interactions) and may take place between neighbours in space or on a specific underlying network. Under some conditions, in such systems various kinds of transitions occur; during these transitions the objects (particles, organisms or even robots) adopt a pattern of behaviour which is nearly completely determined by the collective effects due to the presence of all of the other objects in the system.

Take ferromagnets as an example. These materials can undergo spontaneous magnetisation, in effect because they are made up of a host of "tiny magnets". At relatively high temperatures, these magnets cannot align with each other and the resulting magnetisation is zero. But at a critical temperature the tendency to adopt a common direction suddenly, but continuously takes over from the effects of fluctuations. So, most of the small magnets, assisting each other in a collective manner, point in the same direction, and magnetisation (order) spontaneously appears. Similarly, a group of feeding pigeons randomly oriented on the ground will order themselves into a uniform flock while flying away after a big disturbance.

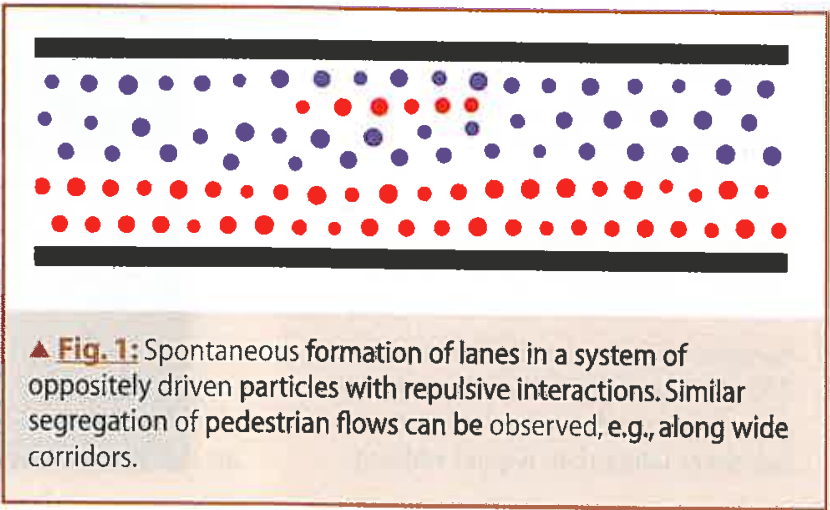




\section{Motivation}

Mankind has been experiencing a long successful period of technological development. This era has been the result of a deeper understanding of the various physical and chemical processes due to the outstanding advances in the related sciences. After these achievements there is now a growing interest in a better, more exact understanding of the mechanisms underlying the main processes in societies as well. There is a clear need for the kind of firm, reliable results produced by natural sciences in the context of the studies of human behaviour. The revolution in information and transportation technology brings together larger and larger masses of people (either physically or through electronic communication). New kinds of communities are formed, including, among many others, internet chat groups or huge crowds showing up at various performances, transportation terminals or demonstrations. Since they represent relatively simple examples, these groups or communities of people provide a good subject from the point of studying the mechanisms determining the phenomena taking place in societies.

In the following I would like to show that the concept of collective phenomena can be applied to the group behaviour of people. In a way understanding a new phenomenon is usually realised by relating it to a known one; a more complex system is understood through analysing its simpler variants. In the seventies there was a major breakthrough in statistical physics when a deep theoretical understanding of a general type of phase transitions was achieved due to the invention of the so called renormalisation group method. This theory showed that the main features of transitions are insensitive to the details of the interaction between the objects in a system, thus, as an extreme case, orientational forces between atoms may result in ordering phenomena similar to those observed in groups of much more complex units.

Below we discuss new quantitative approaches to collective behaviour based on the exact methods of statistical physics. It is clear that the methods developed in natural sciences contain a significantly smaller amount of subjectivity than those used for the interpretation of human behaviour. If more exact approaches could be applied to social situations they could provide the desired objectivity, reproducibility and predictability. We demonstrate that in cases when the interactions between the members of a group are relatively well defined (e.g, pedestrian traffic, rhythmic applause, panic, soccer fans in stadiums, etc) the corresponding numerical models reproduce relevant aspects of the observed phenomena. Simulating models in a computer has the following advantages: i) by changing the parameters different situations can easily be created ii) the results of an intervention can be predicted and iii) more efficient design of the conditions for the optimal outcome can be assisted. In addition to possible applications, our approach is useful in providing a deeper insigh into the details of the mechanisms determining collective phe. nomena occurring in social groups. Most of the results I an discussing next are available through the home page given in Ref [1]. There exist additional remarkable efforts in similar direction by groups working on traffic, evacuation dynamics, econophysics and on further related topics (see, e.g., Refs 2-4).

\section{Methods}

Our central statement is that collective behaviour can be very effi. ciently studied by the methods developed by statistical physicists The related theoretical and numerical approaches provide reliable, sometimes exact description of the processes taking plact in many particle systems. We assume that under some conditions a large group of humans can be considered as a collection of par. ticles, since there are various situations where the interaction 0 people is reasonably well defined (e.g., two people headin towards each other in a corridor will avoid each other just as i they had a repulsive physical force acting between them).

For the last two decades perhaps the most fruitful approach to collective phenomena has been the application of computer simu. lations. In such studies a simple model is constructed which is supposed to grab the most relevant features of the system to be stud. ied. Then, by letting the algorithm run in the computer while monitoring the parameters of the models a great variety of collective phenomena can be observed. The true test of a model is a carefu comparison of its predictions with the behaviour of the real system

\section{Examples}

The rest of the paper will present examples of group behaviour 0 : people which could be successfully interpreted by computer sim. ulations and the related theoretical concepts. It is hoped that the process of simultaneous investigation of particular examples anc the abstraction of their most general features will in time lead to : coherent theoretical description of collective human behaviour.

\section{Collective motion}

Here we first address the more general question whether ther are some global, perhaps universal features of collective motior [5]. Such behaviour takes place when many organisms are simul. taneously moving and parameters like the level of perturbation: or the mean distance between the individuals is changed.

1. One-dimensional case: Let us first consider a simple case of the particles (people) moving along a line (very narrow corridor, sc that two people cannot get around each other). The particles art
Fig. 2 .

a) (left) Snapshot of a simulation in which the particles are moving under the influence of forces mimicking sociopsychological and physical interactions. For large desired velocities (escape situation) jamming occurs at the exit.

b) (right) The time needed for all of the 200 particles to leave the room starts to grow beyond a desired velocity about two times larger than regular walking.
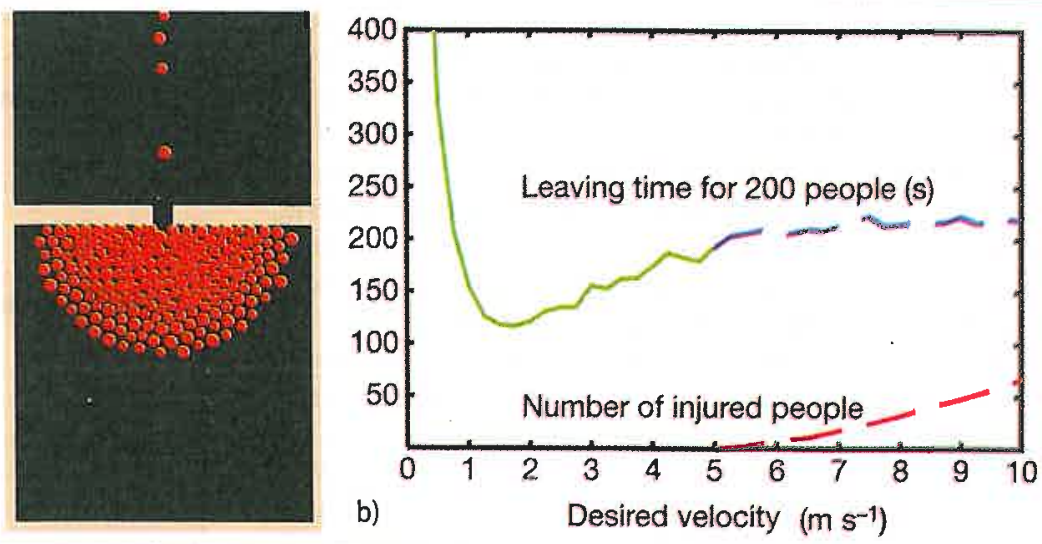


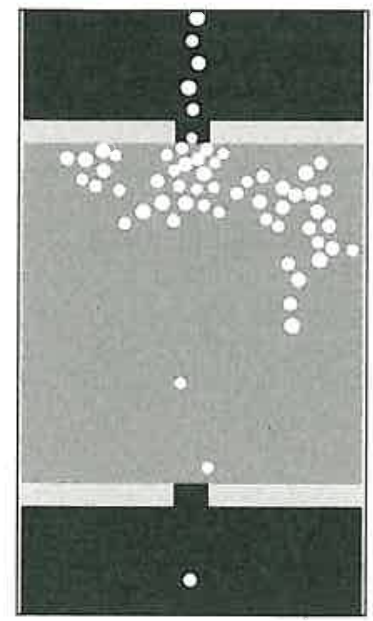

4ig. 3: Snapshot of a simulation with particles which, in addition to the repulsive socio-psychological and physical forces, attempt to follow each other. In this case the particles simulate people trying to escape from a smoky room having two exits. There is an optimal degree of "herding" or cooperation, when medium-sized groups are formed collectively leaving the room. trying to maintain the same speed except when they are forced to turn back. There are only two rules: a) Follow the others, or in other words, try to take on the average velocity of your neighbours (who all move left or right). b) In addition, an amount of randomness is added to the actual velocity (to account for example for the level of excitement of the pedestrians).

Simulations result in a completely disordered motion if the level of perturbations is large (each particle moves back and forth randomly). However, if the noise is smaller than a critical value (just as in the case of the ordering of ferromagnets), groups of particles are spontaneously formed the groups merge (aggregate) and sooner or later join into a single large group moving in a direction determined in a non-trivial way by the initial conditions. In the first, disordered case the people stay "trapped" in the corridor, even in the case of fire, while the ordered motion corresponds to a chance for escaping.

2. Two dimensions: Here the particles can get around each other and the pattern of motion is changed. Nevertheless, using the rules of "follow the others" and the application of perturbations leads to a transition from a disorderly moving crowd to an ordered "flock" when the level of perturbations is lowered.

A) Consider, as a thought experiment, thousands of people standing on a square and trying to look in the same-however, previously undetermined-direction, after being asked to do so. A nice example for human collective behaviour would be if all of them managed to face the same direction. Can they do it? Statistical physicists can predict for sure that this cannot be done. They recall a theorem valid for particles with short ranged ferromagnetic interactions stating that in two dimensions no long range ordered phase (all magnets pointing in the same direction) can exist in such a system for any finite temperature and zero external field. So what happens? Locally people are looking almost in the same direction, but on a large scale, e.g., seen from a helicopter-just as the little magnets-they locally form vortex-like directional patterns due to the small perturbations due to human errors. Curiously enough, if the crowd is allowed to choose from a few discrete directions, the ordering can be realised. Perhaps even more interestingly, our models of flocking (based on the follow the neighbours rule) predict that if the people are asked to move in the same direction they will be able to do it.

B) In the latter models, if the moving particles are confined to move around in a closed circular area stable motion can be maintained only by the simultaneous rotation of all of the objects around the centre. Remarkably enough, under some conditions even humans move in groups in a manner predicted by simple models. Indeed, in Mecca each year thousands of people circle around the Kaba stone as they are trying to both keep on moving and not confronting with others.

C) Next we focus on a system of oppositely moving pedestrians in a corridor. Here the corridor is wide enough (its width is several times larger than the diameter of a person). Half of the pedestrians is assumed to move from left to right, the rest in the opposite direction. In the associated model it is assumed that the particles tend to take on a constant speed in their desired direction and are avoiding each other due to a repulsive force.

Simulations of this simple model based on the solution of the corresponding Newton's equations of motion reproduce the experimentally observed behaviour surprisingly well. A spontaneous formation of lanes of uniform walking directions in "crowds" of oppositely moving particles can be observed (Fig. 1). It is clear that lane formation will maximise the average velocity in the desired walking direction which is a measure of the "efficiency" or "success" of motion. Note, however, that lane formation is not a trivial effect of this model, but eventually arises only due to the smaller relative velocity and interaction rate that pedestrians with the same walking direction have. Once the pedestrians move in uniform lanes, they will have very rare and weak interactions.

\section{Panic}

One of the most disastrous forms of collective human behaviour is the kind of crowd stampede induced by panic, often leading to fatalities as people are crushed or trampled. Sometimes this behaviour is triggered in life-threatening situations such as fires in crowded buildings; at other times, stampedes can arise from the rush for seats or seemingly without causes. Although engineers are finding ways to alleviate the scale of such disasters, their frequency seems to be increasing due to greater mass events. Next we show that simulations based on a model of pedestrian behaviour can provide valuable insights into the mechanisms of and preconditions for panic and jamming by incoordination [6].

The available observations on escape panic have encouraged us to model this kind of collective phenomenon in the spirit of selfdriven many-particle systems. We assume, in addition to the earlier considered socio-psychological forces the relevance of physical forces as well since the latter ones become very important in the case of a dense crowd with strong drive to get through a narrow exit. Each pedestrians of mass $m_{i}$ likes to move with a certain desired speed $v_{i}{ }^{0}(t)$ into a certain direction $\mathrm{e}_{i}{ }^{0}(t)$, and therefore tends to correspondingly adapt his or her actual velocity $v_{i}(t)$ with a certain characteristic time $\tau$. Simultaneously, he or she tries to keep a velocity-dependent distance to other pedestrians $j$ and walls $W$. This can be modelled by "interaction forces" $f_{i j}$ and $f_{i w}$, respectively. In mathematical terms, the change of velocity in time is then given by the acceleration equation

$$
m_{i} d \mathrm{v}_{\mathrm{i}} / d t=m_{i}\left[\mathrm{v}_{i}^{0}(t) \mathrm{e}_{i}^{0}(t)-\mathrm{v}_{i}(t)\right] / \tau+\Sigma_{j} \mathrm{f}_{i j}+\mathrm{f}_{i W}
$$

The $f_{i j}$ interaction forces include an exponentially decaying, repelling term expressing socio-psychological effects, and two additional "physical" terms corresponding to elastic repulsion and friction forces between the bodies of people.

The $f_{i W}$ interaction with the walls is treated analogously. To avoid model artefacts (gridlocks by exactly balanced forces in symmetrical configurations), a small amount of irregularity of almost arbitrary kind is needed. This irregularity was introduced by uniformly distributed pedestrian diameters $r_{i}$ in the interval $[0.5 \mathrm{~m}, 0.7 \mathrm{~m}]$, approximating the distribution of shoulder widths of soccer fans. 
Based on the above model assumptions, it is possible to simulate several important phenomena of escape panic. The simulated outflow from a room is well-coordinated and regular, if the desired velocities are normal. However, for desired velocities above $1.5 \sim \mathrm{m} / \mathrm{s}$, i.e., for people in a rush, we find an irregular succession of arch-like blockings of the exit and avalanche-like bunches of leaving pedestrians, when the arches break. "Fasteris-slower effect" due to impatience: Since clogging is connected with delays, trying to move faster can cause a smaller average speed of leaving, if the friction parameter is large (Fig. 2). This effect is particularly tragic in the presence of fires, where the fleeing people reduce their own chances of survival. Improved outflows can be reached by columns placed asymmetrically in front of the exits preventing the build up of fatal pressures.

Finally, we investigate a situation in which pedestrians are trying to leave a smoky room, but first have to find one of the invisible exits. Each pedestrian may either select an individual direction or follow the average direction of his neighbours in a certain radius or try a mixture of both. We assume that both options are weighted with some parameter (1-p) and $p$, respectively, where $0<p<1$. As a consequence, we have individualistic behaviour if $p$ is low, but herding behaviour $p$ is high. Our model suggests that neither individualistic nor herding behaviour performs well. Pure individualistic behaviour implies that each pedestrian finds an exit only accidentally, while pure herding behaviour implies that the complete crowd is eventually moving into the same and probably blocked direction, so that available exists are not efficiently used, in agreement with observations. Accordingly, we find optimal chances of survival for a certain mixture of individualistic and herding behaviour, where individualism allows some people to detect the exits and herding guarantees that successful solutions are followed by the others (Fig. 3).

\section{Synchronisation}

1. Rhythmic applause: An audience expresses appreciation for a good performance by the strength and nature of its applause. The initial thunder often turns into synchronised clapping - an event familiar to many who frequent concert halls. Synchronised clapping has a well defined scenario: the initial strong incoherent clapping is followed by a relatively sudden synchronisation process, after which everybody claps simultaneously and periodically. This synchronisation can disappear and reappear several times during the applause. The phenomenon is a delightful expression of social self-organisation, that provides a human scale example of the synchronisation processes observed in numerous systems in nature [7].

The above scenario can be recorded and the recordings analysed using the techniques common in physics [8]. The analysis reveals various interesting features including a spontaneous period doubling (as compared to the natural period of a single spectator) when the synchronisation takes place. In other words, after an initial asynchronous phase, characterised by high frequency clapping (Mode I), the individuals synchronise by eliminating every second beat, suddenly shifting to a clapping mode with double period (Mode II) where the dispersion (the relative difference in the clapping frequencies) is smaller. Statistical theories developed for a group of globally coupled periodically behaving objects can be used to demonstrate that the necessary condition for synchronisation is that dispersion has to be smaller than a critical value. Consequently, period doubling emerges as a condition of synchronisation, since it leads to slower clapping modes during which significantly smaller dispersion can be maintained. Thus, the evaluation of the measurements offers a key

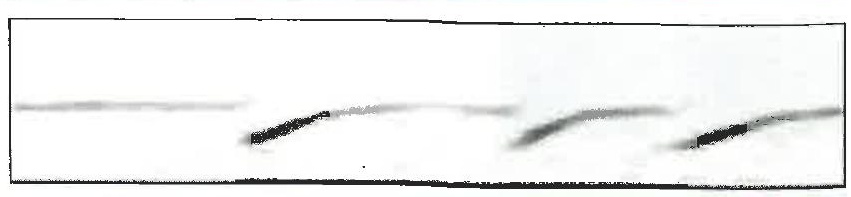

Aig. 4: Fourier-gram of the clapping sound intensity after a great performance in one of the many theatres in Budapest. Grey level indicates the amplitude of the Fourier spectrum for the given frequency. Time is shown along the horizontal axis. The occurrence of the doubling and the subsequent decrease of the global period is indicated to happen several times.

insight into the mechanism of synchronised clapping: fast clapping synchronisation is not possible due to the large dispersion in the clapping frequencies. After period doubling, as Mode II clapping with small dispersion appears, synchronisation can be and is achieved. However, as the audience gradually decreases the period to enhance the average noise intensity, it gradually slips back to the fast clapping mode with larger dispersion, destroying synchronisation (Fig. 4).

2. Mexican wave, becoming widely known during the 1986 World Cup held in Mexico, has since become a favourite paradigm for a variety of systems in which an initial perturbation propagates in the form of a single "planar" wave. The most important reason for the increasing popularity of this phrase, also known as La Ola, (or simply "the wave"), is likely to be due to its unique origin; it means a human wave moving along the stands of stadia as one section of spectators stands up, arms lifting, then sits as the next section does the same.

Using video recordings we have analysed 14 waves in stadiums with above 50.000 people: the wave has a typical velocity in the range of $12 \mathrm{~m} / \mathrm{s}$ (20 seats $/ \mathrm{s})$, a width of about $6-12 \mathrm{~m}(\sim 15$ seats) and more frequently rolls in the clockwise direction [9]. It is generated by the simultaneous standing up of not more than a few dozens of people and subsequently expands over the entire tribune acquiring its stable, close to linear shape. (see page $\mathrm{http}: / /$ angel.elte.hu/wave) dedicated to this research, offering further data and interactive simulations).

The relative simplicity of the Mexican wave allows us to develop a quantitative treatment of this kind of collective behaviour by building and simulating models accurately reproducing and

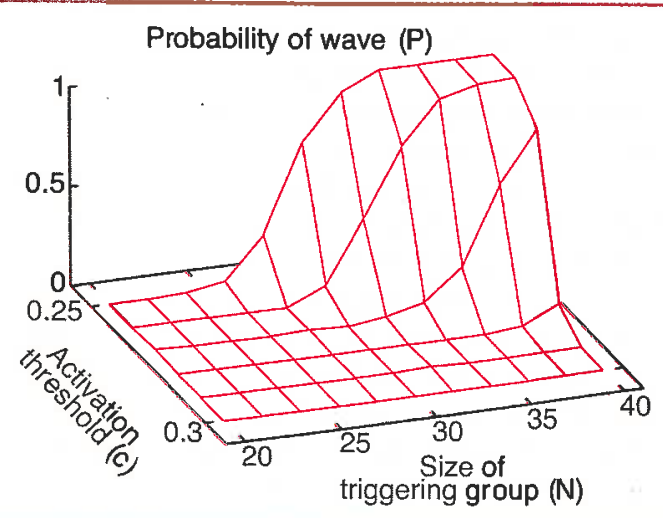

4. Fig. 5 This figure shows the probability of generating a Mexican wave as a function of the number of the initiators and a parameter $c$ corresponding to the critical level of excitement beyond which a person becomes active. 
predicting the details of the associated human wave. It can be shown that the well established approaches to the theoretical interpretation of excitable media-originally created for describing such processes as forest fires or wave propagation in heart tissue-can readily be generalised to include human social behaviour. In analogy with models of excitable media, people are regarded as excitable units: they can be activated by an external stimulus (a distance and direction-wise weighted concentration of nearby active people exceeding a threshold value). Once activated, each unit follows the same set of internal rules to pass through the active (standing and waving) and refractory (passive) phases before returning to its original, resting (excitable) state.

Next, we employed these models to get an insight into the conditions for triggering a wave (Fig. 5). Our results clearly demonstrate that the dependence of the eventual occurrence of a wave on the number of initiators is a rather sharply changing function, i.e., triggering a Mexican wave requires a critical mass. This approach is expected to have implications for the treatment of situations where influencing the behaviour of a crowd is desirable. In particular, in the context of violent street incidents associated with demonstrations or sport events, it is essential to know under what conditions groups can get control over the crowd and how fast and in which form this perturbation/transition can spread.

\section{Conclusions}

The models of collective behaviour of humans can account for a number of specific features of social behaviour under certain conditions. The advantage of the models is that by changing the parameters different situations can be easily created. Models adequately describing group phenomena can be used for predictions. In addition to such more concrete possible applications of simula- tions as the design of escape routes or better networks, the models are useful in providing a deeper insight into the mechanisms behind such collective phenomena as synchronisation or panic.

\section{Acknowledgement}

The investigations briefly described above have been carried out in cooperation with a number of colleagues. Here I would like to thank my principal collaborators, A-L. Barabási, A. Czirhe models are useful in providing a dee

An edited version of a talk delivered at EPS12, Budapest, August 2002

\section{References}

[1] http://angel.elte.hu/ vicsek

[2] D. Helbing, Traffic and related self-driven many particle systems, (2001) Rev. Mod. Phys., 73, 1067-1141

[3] R. Mantegna and H. E. Stanley, An introduction to econophysics, (2001) Cambridge University Press, Cambridge

[4] M.Schreckenberg and S. D. Sarma. Pedestrian and Evacuation Dynamics (PED), (2001), Springer, Berlin

[5] Vicsek, T. (2001) ed. Scaling and Fluctuations in Biology, Oxford University Press, Oxford

[6] D. Helbing, I. Farkas. and T. Vicsek, T. (2000) Simulating dynamical features of escape panic Nature 407, 487-490

[7] A. P. Pikovsky, M. Rosemblum and J. Kurths, Synchronisation, (2001) Cambridge University Press, Cambridge

[8] Z. Néda, Ravasz, E., Y. Brechet, T. Vicsek and A-L. Barabási, (2000) The sound of many hands clapping Nature, 403, 849-851

[9] I. Farkas, D. Helbing and T. Vicsek, (2002), Mexican waves in an excitable medium Nature 419, 131-132

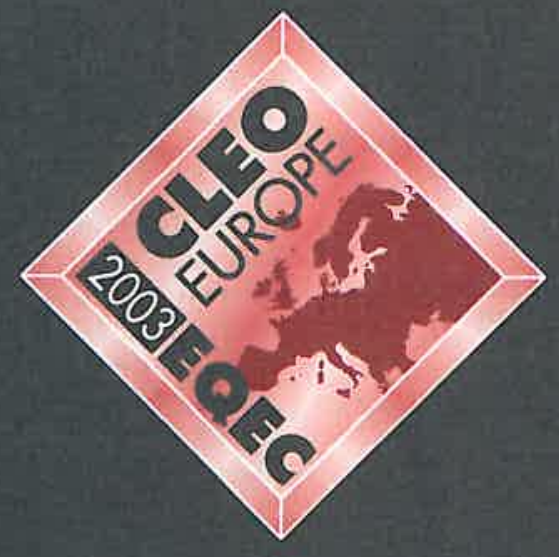

22-27 June 2003 Munich, Germany

Conference Secretariat

BP2136, F 68060 Mathour Ced

tel: +33389329442 fax: +333893294 ag

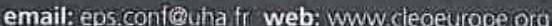

CLEO $/$ Europe-EQEC 2003 is Europe's premier joint conference on lasers, electrooptics and fundamental quantum electronics.

The conference will be held at the Munich International Congress Centre in conjunction with Laser 2003, the largest European exhibition of laser and electro-optic equipment and services.

CLEO $/$ Europe-EQEC 2003 is the place to discover the latest research developments, applications, and technology in the field

\section{Online registration now open}

www.cleoeurope.org 